\title{
Computing Homomorphism Spaces between Modules over Finite Dimensional Algebras
}

\author{
Klaus M. Lux and Magdolna Szőke
}

\section{CONTENTS}

1. Introduction

2. Homomorphisms and Presentations

3. The Generators

4. The Defining Relations

5. The Homomorphisms

6. The Implementation

Acknowledgments

References

2000 AMS Subject Classification: Primary 16Z05; Secondary 16G99, 20C40

Keywords: Homomorphism spaces, algorithm, algebras
We describe an algorithm to compute homomorphism spaces between modules of finite dimensional algebras over finite fields. The algorithm is implemented in the C-Meat-Axe.

\section{INTRODUCTION}

In this paper, we study the fundamental algorithmic problem of determining the homomorphisms from an $A$ module $M$ to an $A$-module $N$ over an algebra $A$. We shall outline an algorithm for the case that $A$ is a finite dimensional algebra over a finite field $F$ and both modules $M$ and $N$ are finitely generated as $A$-modules.

More specifically, the input to our algorithm consists of the following data: The modules $M$ and $N$ are given in terms of linear algebra via the matrices for a generating system of the $F$-algebra $A$. Note that this is a common situation in the study of representations for a given finite dimensional algebra. The output of the algorithm will be matrices that form an $F$-basis for the $F$-vector space of $A$-homomorphisms from $M$ to $N$. For a different set-up in the case that $A$ is commutative, see the end of the introduction.

In case $M=N$, we have determined an $F$-basis of the endomorphism ring of $M$. One of the reasons for focusing on the endomorphism ring of a module is given by the fact that we can use the knowledge about the structure of the endomorphism ring to give a decomposition of $M$ into indecomposable $A$-modules. In a separate paper, we shall give an algorithm that determines such a decomposition of $M$ (see [Lux and Szőke 03]).

As a starting point of our investigation of a homomorphism space from an $A$-module $M$ to an $A$-module $N$, we make use of well-known algorithms that can answer structural questions about a given module. For example, the composition factors, the socle, and the radical series can be determined algorithmically; see [Parker 84], [Lux

(c) A K Peters, Ltd $1058-6458 / 2001 \$ 0.50$ per page Experimental Mathematics 12:1, page 91 
and Wiegelmann 01], [Szőke 98]. Along these lines, the algorithm for calculating homomorphism spaces can be seen as a natural extension in the study of the category of finite dimensional $A$-modules from the algorithmic point of view.

A first implementation of an algorithm for calculating homomorphism spaces in the computer algebra system CAYLEY was described by G. J. A. Schneider; see [Schneider 90]. The algorithm outlined by Schneider is derived straightforwardly from the definition of $A$ homomorphisms. Its major drawback is its poor performance, which is mainly due to the fact that the algorithm did not take advantage of the given module structures nor the algorithms mentioned above. Again we emphasize that one should first determine the module structures and then use this information to determine a basis of the homomorphism space in question.

A similar line of thought to ours has been used by C. Leedham-Green. He has developed an algorithm together with J. Cannon, whose details are unpublished. This algorithm is part of the computer algebra system MAGMA (see [Bosma and Cannon 99]), and replaces Schneider's original approach. Comparing the GAP-implementation of Leedham-Green's algorithm by M. Smith with our algorithm, one can see that our algorithm differs from Leedham-Green's in several places. First of all, we do not use a random strategy for generating words in $A$ but use so-called peakwords related to the composition factors (see [Lux et al. 94]) for constructing module generators. Secondly, we use a different method for processing the system of linear equations whose solutions give the homomorphisms. This enables us to determine the number of unknowns and the number of equations explicitly; see the end of Section 3. Table 1 at the end of Section 6. should convince the reader that in most cases our approach is better than the one in MAGMA.

We want to remark that the problem of determining endomorphism rings and homomorphism spaces is also of interest in commutative algebra. The setting there is slightly different, since the algebras of main interest will be multivariate polynomial rings and their quotient rings. In this case, the algebra in question is infinite dimensional and the modules under consideration also tend to be infinite dimensional. This disadvantage, however, is accompanied by the advantage that homomorphism spaces are again modules over the polynomial ring. Furthermore, the modules and algebras are usually given as finitely presented objects, i.e., by a finite set of generators and relations. This alternative description replaces the description of a module in terms of matrices for generators of $A$; see above. The idea of working with presentations of the modules turns out to be very useful in our context, too. Roughly speaking, homomorphisms are easier to determine if the module $M$ is finitely presented. Actually, it is well known (see Lemma 2.1) that the matrices describing a module can also be interpreted as giving a presentation for the module. Since the algebras we study are usually noncommutative, we cannot give a presentation for the homomorphism space, but we have to describe it as an $F$-vector space. Note that C. Struble generalised the approach for commutative algebras to the case of algebras given by a quiver with relations; see [Green et al. 01].

\section{HOMOMORPHISMS AND PRESENTATIONS}

In the following, we fix a finite field $F$ and a finite dimensional $F$-algebra $A$. Let $M$ and $N$ be finitely generated right $A$-modules. The object we want to study is the $F$-vector space of $A$-homomorphisms from $M$ to $N$ :

$$
\begin{aligned}
\operatorname{Hom}_{A}(M, N)= & \{\varphi: M \rightarrow N \mid \varphi \text { is } F \text {-linear, } \\
& (m a) \varphi=(m \varphi) a \text { for all } m \in M, a \in A\} .
\end{aligned}
$$

More precisely, we describe an algorithm that determines an $F$-basis of $\operatorname{Hom}_{A}(M, N)$. In the following, we assume that the modules $M$ and $N$ are given in terms of matrices for a fixed $F$-algebra generating system $a_{1}, \ldots$, $a_{\text {ngen } A}$ of $A$.

Let $X$ be an $A$-module and let

$$
\Phi: X \rightarrow M
$$

be an $A$-epimorphism. Then each $A$-homomorphism $\varphi: M \rightarrow N$ gives rise to an $A$-homomorphism $\Phi \varphi: X \rightarrow$ $N$. Conversely, a homomorphism $\psi \in \operatorname{Hom}_{A}(X, N)$ is of the form $\Phi \varphi$ for an $A$-homomorphism $\varphi: M \rightarrow N$ if and only if $\psi$ restricted to $\operatorname{Ker} \Phi$ is the zero map. Hence, we have an injective $F$-linear map,

$$
\Phi^{*}: \operatorname{Hom}_{A}(M, N) \longleftrightarrow \operatorname{Hom}_{A}(X, N), \quad \varphi \mapsto \Phi \varphi,
$$

with

$$
\operatorname{Im} \Phi^{*}=\left\{\psi \in \operatorname{Hom}_{A}(X, N) \mid(\operatorname{Ker} \Phi) \psi=0\right\} .
$$

Using $\Phi^{*}$, we can and do identify $\operatorname{Hom}_{A}(M, N)$ with the subspace $\operatorname{Im} \Phi^{*}$ of $\operatorname{Hom}_{A}(X, N)$.

Let $X=\mathcal{F}\left(x_{1}, \ldots, x_{r(X)}\right)$, a free $A$-module of rank $r(X)$ on the generators $x_{1}, \ldots, x_{r(X)}$, and let $\left[n_{1}, \ldots\right.$, 
$\left.n_{\operatorname{dim} N}\right]$ be a basis of $N$. Define the $A$-homomorphisms $\psi_{i, j}: X \rightarrow N$ for $1 \leqslant i \leqslant r(X), 1 \leqslant j \leqslant \operatorname{dim} N$ by

$$
x_{k} \psi_{i, j}= \begin{cases}n_{j} & \text { if } i=k \\ 0 & \text { if } i \neq k\end{cases}
$$

Then the elements $\psi_{i, j}$ (for $1 \leqslant i \leqslant r(X), 1 \leqslant j \leqslant$ $\operatorname{dim} N)$ form a basis of $\operatorname{Hom}_{A}(X, N)$. Therefore, each $A$ homomorphism $\psi: X \rightarrow N$ can be expressed uniquely as an $F$-linear combination

$$
\psi=\sum_{i=1}^{r(X)} \sum_{j=1}^{\operatorname{dim} N} c_{i, j} \psi_{i, j}
$$

Assume

$$
y=\sum_{k=1}^{r(X)} x_{k} b_{k} \in \operatorname{Ker} \Phi,
$$

where $b_{k} \in A$ for all $1 \leqslant k \leqslant r(X)$. Recall that $\psi \in \operatorname{Hom}_{A}(M, N)$ if and only if it vanishes on $\operatorname{Ker} \Phi$. Therefore,

$$
\begin{aligned}
0=y \psi & =\left(\sum_{k=1}^{r(X)} x_{k} b_{k}\right)\left(\sum_{i=1}^{r(X)} \sum_{j=1}^{\operatorname{dim} N} c_{i, j} \psi_{i, j}\right) \\
& =\sum_{i, j, k} c_{i, j}\left(x_{k} \psi_{i, j}\right) b_{k} \\
& =\sum_{i, j} c_{i, j}\left(n_{j} b_{i}\right) .
\end{aligned}
$$

Let $M\left(b_{i}\right)$ (for $1 \leqslant i \leqslant r(X)$ ) be the matrix of $b_{i}$ with respect to the basis $\left[n_{1}, \ldots, n_{\operatorname{dim} N}\right]$ of $N$. Then the above equality is equivalent to

$$
\sum_{i=1}^{r(X)} \sum_{j=1}^{\operatorname{dim} N} c_{i, j} M\left(b_{i}\right)_{j, k}=0
$$

for all $k=1, \ldots, \operatorname{dim} N$. If the coefficients $c_{i, j}$ are considered to be unknowns, the above equalities give $\operatorname{dim} N$ linear equations in these unknowns. Note that it is sufficient to consider the equations derived from an $A$-module generating system of $\operatorname{Ker} \Phi$ in order to obtain a system of linear equations in the coefficients $c_{i, j}$ whose solutions are exactly the coefficients describing $A$-homomorphisms $\psi: M \rightarrow N$.

We are therefore interested in epimorphisms $\Phi$ from free modules onto $M$ and generating systems for the kernels of such epimorphisms $\Phi$. As above, let $\mathcal{F}\left(x_{1}, \ldots\right.$, $\left.x_{\text {ngen } M}\right)$ be a free $A$-module of rank ngen $M$ on the generators $x_{1}, \ldots, x_{\text {ngen } M}$. We define a presentation of $M$ as an epimorphism $\Phi: \mathcal{F}\left(x_{1}, \ldots, x_{\text {ngen } M}\right) \rightarrow M$ together with a generating system $y_{1}, \ldots, y_{\text {nrel } M}$ for $\operatorname{Ker} \Phi$.
Note that $\Phi$ is uniquely determined by the images $m_{i}=x_{i} \Phi$ for $i=1, \ldots$, ngen $M$ and that these images form a generating system of the $A$-module $M$. Therefore, giving a presentation of $M$ is equivalent to giving a generating system $m_{1}, \ldots, m_{\text {ngen } M}$ of $M$ and a system $\sum x_{i} b_{i, j}$ for $1 \leqslant j \leqslant \operatorname{nrel} M, b_{i, j} \in A$, generating the kernel of $\Phi$.

By a slight abuse of notation we use the following to denote a presentation of $M$ :

$$
\left\langle x_{1}, \ldots, x_{\text {ngen } M} \mid \sum_{i=1}^{\text {ngen } M} x_{i} b_{i, j}=0, \quad 1 \leqslant j \leqslant \operatorname{nrel} M\right\rangle .
$$

In this notation, the equations $\sum_{i=1}^{\text {ngen } M} x_{i} b_{i, j}=0$ are called defining relations with respect to the generators $x_{1}, \ldots, x_{\text {ngen } M}$.

Let $\mathfrak{F}$ be the free $F$-algebra of rank ngen $A$ on the generators $\mathfrak{f}_{1}, \ldots, \mathfrak{f}_{\text {ngen } A}$. Then we have a well-defined $F$-algebra epimorphism $\pi: \mathfrak{F} \rightarrow A$ defined by $\mathfrak{f}_{i} \mapsto a_{i}$ for $1 \leqslant i \leqslant \operatorname{ngen} A$. Moreover, the $A$-module $M$ can be considered as an $\mathfrak{F}$-module by inflation. Let

$$
\left\langle x_{1}, \ldots, x_{\text {ngen } M} \mid \sum_{i=1}^{\text {ngen } M} x_{i} \mathfrak{b}_{i, j}=0, \quad 1 \leqslant j \leqslant \operatorname{nrel} M\right\rangle
$$

be a presentation of $M$ as an $\mathfrak{F}$-module, where $\mathfrak{b}_{i, j}$ are elements of $\mathfrak{F}$ for all $1 \leqslant i \leqslant n g e n A$ and $1 \leqslant j \leqslant \operatorname{nrel} M$. Denote the images $\mathfrak{b}_{i, j} \pi \in A$ by $b_{i, j}$. Then

$$
\left\langle x_{1}, \ldots, x_{\text {ngen } M} \mid \sum_{i=1}^{\text {ngen } M} x_{i} b_{i, j}=0, \quad 1 \leqslant j \leqslant \operatorname{nrel} M\right\rangle
$$

is certainly a presentation of $M$ as an $A$-module.

The presentations of $A$-modules we are giving in the sequel will all come from presentations as $\mathfrak{F}$-modules. One of the reasons is that we do not know the algebra $A$ itself, just its image $A / \operatorname{Ann}(M)$, where $\operatorname{Ann}(M)$ denotes the annihilator ideal of $M$. However, it is not enough to give a presentation of $M$ as an $A / \operatorname{Ann}(M)$-module since $N$ need not be annihilated by $\operatorname{Ann}(M)$. Note that $\operatorname{Hom}_{A}(M, N)=\operatorname{Hom}_{\mathfrak{F}}(M, N)$.

As the following well-known lemma shows, an $F$ matrix representation of $A$ describing the action of $A$ on the $A$-module $M$ can also be used to define a presentation of $M$.

Lemma 2.1. Suppose that the A-module $M$ is given in terms of matrices $M_{B}\left(a_{i}\right)$ for the $F$-algebra generating system $a_{1}, \ldots, a_{\text {ngen } A}$ of $A$ with respect to the $F$-basis $B=\left[m_{1}, \ldots, m_{\operatorname{dim} M}\right]$ of $M$. Then the following gives a 
presentation of $M$ as an $\mathfrak{F}$-module and hence, also as an A-module:

$$
\begin{array}{r}
\left\langle x_{1}, \ldots, x_{\operatorname{dim} M}\right| x_{j} \cdot \mathfrak{f}_{i}-\sum_{k=1}^{\operatorname{dim} M} M_{B}\left(a_{i}\right)_{j, k} x_{k}=0 ; \\
1 \leqslant i \leqslant \operatorname{ngen} A, 1 \leqslant j \leqslant \operatorname{dim} M\rangle .
\end{array}
$$

Proof: Denote by $\mathcal{F}\left(x_{1}, \ldots, x_{\operatorname{dim} M}\right)$ a free $\mathfrak{F}$-module of rank $\operatorname{dim} M$ on the generators $x_{1}, \ldots, x_{\operatorname{dim} M}$. Moreover, define the $\mathfrak{F}$-submodule $K$ of $\mathcal{F}\left(x_{1}, \ldots, x_{\operatorname{dim} M}\right)$ as follows:

$$
\begin{array}{r}
K=\left\langle x_{j} \mathfrak{f}_{i}-\sum_{k=1}^{\operatorname{dim} M} M_{B}\left(a_{i}\right)_{j, k} x_{k}\right| 1 \leqslant i \leqslant \operatorname{ngen} A, \& \\
1 \leqslant j \leqslant \operatorname{dim} M\rangle_{\mathfrak{F}} .
\end{array}
$$

In order to prove that we get a presentation for $M$, we have to show that $K$ is the kernel of the $\mathfrak{F}$-epimorphism $\Phi: \mathcal{F}\left(x_{1}, \ldots, x_{\operatorname{dim} M}\right) \rightarrow M$ mapping $x_{i}$ to $m_{i}$. Equivalently, $\Phi$ induces an $\mathfrak{F}$-isomorphism between $M$ and $Q:=\mathcal{F}\left(x_{1}, \ldots, x_{\operatorname{dim} M}\right) / K$. Since $K$ is in the kernel of $\Phi$, we get a well-defined epimorphism from $Q$ onto $M$. By the definition of $K$, the set $x_{i}+K$ (for $i=1, \ldots$, $\operatorname{dim} M)$ is an $F$-generating set for $Q$, so it follows that $M$ is isomorphic to $Q$ as $\mathfrak{F}$-modules.

To compute the homomorphism space $\operatorname{Hom}_{\mathfrak{F}}(M, N)=$ $\operatorname{Hom}_{A}(M, N)$ more efficiently, we are interested in reducing the number of generators in a given presentation of $M$. The following lemma deals with a setting in which this can be achieved.

Lemma 2.2. Let $\left[m_{1}, \ldots, m_{\text {ngen } M}\right]$ be an A-generating system for $M$, linearly independent over $F$. Extend this sequence to a basis $B=\left[m_{1}, \ldots, m_{\operatorname{dim} M}\right]$ of $M$ and for $j>$ ngen $M$ express $m_{j}$ as an $\mathfrak{F}$-linear combination $\sum_{i=1}^{\text {ngen } M} m_{i} w_{i, j}$. Define the elements $z_{j} \in \mathcal{F}\left(x_{1}\right.$, $\left.\ldots, x_{\text {ngen } M}\right)$ as $x_{j}$ for $j=1, \ldots$, ngen $M$ and as $\sum_{i=1}^{\text {ngen } M} x_{i} w_{i, j}$ for $j=\operatorname{ngen} M+1, \ldots, d$. Then the following is a presentation for $M$ :

$$
\begin{aligned}
\left\langle x_{1}, \ldots, x_{\text {ngen } M}\right| z_{j} \mathfrak{f}_{i}-\sum_{k=1}^{\operatorname{dim} M} M_{B}\left(a_{i}\right)_{j, k} z_{k}=0 ; \\
1 \leqslant i \leqslant \operatorname{ngen} A, 1 \leqslant j \leqslant \operatorname{dim} M\rangle .
\end{aligned}
$$

Proof: This follows easily from Lemma 2.1.

In Section 2, we describe the way we choose a generating system $m_{1}, \ldots, m_{\text {ngen } M}$ of $M$. Assume for the moment that we have elements $w_{i} \in \mathfrak{F}$ such that $m_{i} w_{i}=0$.
This enables us to modify our original approach for determining the homomorphism space $\operatorname{Hom}_{A}(M, N)=$ $\operatorname{Hom}_{\mathfrak{F}}(M, N)$ as follows.

Choose bases $\left[n_{i, 1}, \ldots, n_{i, d_{i}}\right]$ of $\operatorname{Ker}_{N}\left(w_{i}\right)$ for $1 \leqslant$ $i \leqslant$ ngen $M$. Define the $\mathfrak{F}$-homomorphisms $\psi_{i, j} \in$ $\operatorname{Hom}_{\mathfrak{F}}\left(\mathcal{F}\left(x_{1}, \ldots, x_{\text {ngen } M}\right), M\right)$ for $1 \leqslant j \leqslant d_{i}, 1 \leqslant i \leqslant$ ngen $M$ by

$$
x_{k} \psi_{i, j}= \begin{cases}n_{i, j} & \text { if } i=k \\ 0 & \text { if } i \neq k .\end{cases}
$$

Let $\psi$ be an arbitrary element of $\operatorname{Hom}_{\mathfrak{F}}(M, N)=\operatorname{Im} \Phi^{*}$. Since $x_{i} \psi \in \operatorname{Ker}_{N}\left(w_{i}\right)$, we can express $\psi$ uniquely as a linear combination of the elements $\psi_{i, j}$ :

$$
\psi=\sum_{i=1}^{\text {ngen }} \sum_{j=1}^{d_{i}} c_{i, j} \psi_{i, j} .
$$

Let

$$
y=\sum_{i=1}^{\text {ngen } M} x_{i} b_{i} \in \operatorname{Ker} \Phi,
$$

where all $b_{i} \in \mathfrak{F}$. Then $\psi$ vanishes on $y$. A simple computation shows that

$$
y \psi=\sum_{i=1}^{\text {ngen } M} \sum_{j=1}^{d_{i}} c_{i, j}\left(n_{i, j} b_{i}\right)=0 .
$$

Let $C=\left[n_{1}, \ldots, n_{\operatorname{dim} N}\right]$ be a basis of $N$ and let $M_{C}\left(b_{i}\right)$ be the matrix of the action of $b_{i}$ on $N$ with respect to $C$. Express the elements $n_{i, j}$ in terms of $C$ :

$$
n_{i, j}=\sum_{k=1}^{\operatorname{dim} N} \lambda_{i, j}^{k} n_{k}
$$

for $1 \leqslant i \leqslant \operatorname{ngen} M$ and $1 \leqslant j \leqslant d_{i}$. Then

$$
n_{i, j} b_{i}=\sum_{k=1}^{\operatorname{dim} N} \lambda_{i, j}^{k} n_{k} b_{i}
$$

and the equality (2-1) leads to the following $\operatorname{dim} N$ equations,

$$
\sum_{i=1}^{\operatorname{ngen} M} \sum_{j=1}^{d_{i}} c_{i, j} \sum_{k=1}^{\operatorname{dim} N} \lambda_{i, j}^{k} M\left(b_{i}\right)_{k, \ell}=0
$$

for $\ell=1, \ldots, \operatorname{dim} N$ in the unknown coefficients $c_{i, j}$. In Section 3., we show that a clever choice of the elements $w_{i}$ leads to a drastic reduction in the number of unknown coefficients in comparison to the approach mentioned in Lemma 2.1.

As before, letting $y$ run through a generating system of $\operatorname{Ker} \Phi$, this gives rise to a basis of $\operatorname{Hom}_{\mathfrak{F}}(M, N)=$ $\operatorname{Hom}_{A}(M, N)$. 


\section{THE GENERATORS}

The generators for the $A$-module $M$ will be derived from generators for $M / J(M)$, where $J(M)$ denotes the radical of $M$. In [Lux and Wiegelmann 01], an algorithm for determining generators for the socle of a module were described, and the method for getting a generating set for $M / J(M)$ follows a very similar line of thought. Again, as in [Lux and Wiegelmann 01], we use peakwords, which are easily produced using the C-Meat-Axe program pwkond.

First, we recall the definition of a peakword from [Lux et al. 94]:

Definition 3.1. Let $S, S_{1}, \ldots, S_{t}$ be a set of pairwise nonisomorphic simple $A$-modules. An element $w$ of $A$ is called a peakword of $A$ for $S$ with respect to $S, S_{1}, \ldots$, $S_{t}$ if the following conditions are satisfied:

(i) $\operatorname{Ker}_{S_{i}}(w)=0$ for every $1 \leqslant i \leqslant t$,

(ii) $\operatorname{dim}_{F} \operatorname{Ker}_{S}\left(w^{2}\right)=\operatorname{dim}_{F} \operatorname{End}_{A}(S)$.

Assume $w$ is a peakword for $S$ with respect to $S, S_{1}$, $\ldots, S_{t}$. Then there is an integer $\ell$ such that $\operatorname{Ker}_{M}\left(w^{\ell}\right)=$ $\operatorname{Ker}_{M}\left(w^{\ell+1}\right)$. We call the subspace $\operatorname{Ker}_{M}\left(w^{\ell}\right)$ the stable kernel of the peakword $w$ and denote it $\operatorname{by~}^{\operatorname{StKer}_{M}}(w)$.

If moreover $S, S_{1}, \ldots, S_{t}$ is a complete set of representatives of the isomorphism classes of the simple $A$-modules, then $w^{k}$ is an idempotent in $A$ for some natural number $k$ since $F$ is finite. Furthermore, $e=$ $1_{A}-w^{k}$ is a primitive idempotent with $S e \neq 0$ and $M e=\operatorname{StKer}_{M}(w)$.

Using the same idea as in [Lux and Wiegelmann 01], we can give a straightforward algorithm based on peakwords for determining generating sets for both $M / J(M)$ and $M$.

Theorem 3.2. Let $S_{1}, \ldots, S_{\mathrm{ncf} M}$ be a complete set of representatives of the composition factors of the A-module M. Moreover, let $w_{1}, \ldots, w_{\text {ncf } M}$ be corresponding peakwords.

Choose an F-linearly independent set of vectors in each $\operatorname{StKer}_{M}\left(w_{i}\right)$ whose cosets modulo $J(M) \cap$ $\operatorname{StKer}_{M}\left(w_{i}\right)$ form a basis of the quotient space. Then these cosets generate the homogeneous component corresponding to $S_{i}$ of $M / J(M)$ and we choose a maxi-

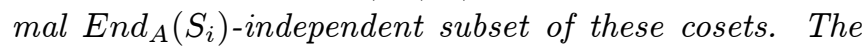
cosets of the selected vectors still generate the homogeneous component, and the union of these sets for all the composition factors is a generating set of $M$.

\section{THE DEFINING RELATIONS}

In Section 3, we described how to determine a generating system for an $A$-module $M$. In this section, we give an algorithm that derives defining relations given a generating system $m_{1}, \ldots, m_{\text {ngen } M}$ of $M$.

It follows Lemma 2.2 and constructs a basis for $M$ as required by the lemma. In the following, this basis will be called a spinning basis of the module $M$. The algorithm also determines a description of the basis elements in terms of the generators $m_{1}, \ldots, m_{\text {ngen } M}$ of $M$ and the generators $a_{1}, \ldots, a_{\text {ngen } A}$ of $A$.

\section{Algorithm 4.1. (Spinning.)}

INPUT: Matrices for an algebra generating system $a_{1}$, $\ldots, a_{\mathrm{ngen} A}$ of $A$ describing the action of the generators on the $A$-module $M$; and an $A$-module generating system $m_{1}, \ldots, m_{\text {ngen } M}$ of $M$.

CALCULATION:

- $B(0):=[]$.

- For $i=1, \ldots$, ngen $M$ do

- $B(i):=B(i-1)$

- If $m_{i} \notin\langle B(i-1)\rangle$ then

* Add $m_{i}$ to $B(i)$.

- Fi.

- For all $m \in B(i) \backslash B(i-1)$ do

* For all $j=1, \ldots$, ngen $A$ do

+ If $m a_{j}$ does not lie in the span of $B(i)$, then

- Add $m a_{j}$ to $B(i)$.

- Store the position of $m$ in $B(i)$ and $j$ as a

description of the new basis element $m a_{j}$.

+ Else

- Write $m a_{j}$ as a linear combination of the

elements of $B(i)$.

- Store the coefficients, which describe a defin-

ing relation of $M$.

$$
+ \text { Fi. }
$$

* End for.

- End for.

- End for. 
OUTPUT: A spinning basis $B$ (ngen $M)$ of $M$, and defining relations of $M$.

Note that the outer "for" loop at a given value of $i$ in Algorithm 4.1 can be executed without knowing the generators $m_{i+1}, \ldots, m_{\text {ngen } M}$. This enables us to look for the next generator $m_{i+1}$ of $M$ after having built up the $i$-th part $B(i)$ of the spinning basis. We call the outer "for" loop of Algorithm 4.1 at the value $i$ the $i$-th part of the spinning algorithm.

Remark 4.2. Since $A$ is generated by $a_{1}, \ldots, a_{\text {ngen } A}$ as an $F$-algebra, and $M$ is generated by $m_{1}, \ldots, m_{\text {ngen } M}$ as an $A$-module, the spinning basis spans the whole module $M$ over $F$, so it is really an $F$-basis of $M$. Moreover, it can be obtained in finitely many steps since $M$ is finite dimensional.

Note that by Lemma 2.1, we obtain a presentation of $M$. Moreover, by Lemma 2.2, the defining relations involve the $A$-generating system $m_{1}, \ldots, m_{\text {ngen } M}$ of $M$.

The number of relations produced by the algorithm is given by

$$
\operatorname{dim}_{F} M \cdot \operatorname{ngen} A-\operatorname{dim}_{F} M+\operatorname{ngen} M .
$$

Indeed, for each $m$ in the spinning basis and each generator $a_{j}$ of $A$, we either have a relation, or a new element of the spinning basis. The latter case occurs $\operatorname{dim} M-\operatorname{ngen} M$ times, so the number of relations is given by the above expression.

If we input a generating system as described in Theorem 2.3, the number of generators of $M$ equals the composition length $\operatorname{cl}(M / J(M))$ of $M / J(M)$, so the number of relations is

$$
\operatorname{dim}_{F} M \cdot \text { ngen } A-\operatorname{dim}_{F} M+\operatorname{cl}(M / J(M)) .
$$

Since each relation describes $\operatorname{dim}_{F} N$ equations, the number of equations is $\operatorname{dim}_{F} N$ times the number of relations.

Note that Algorithm 4.1 does not make use of the fact that our generators are chosen in stable kernels of peakwords. However, this choice of generators gives us a few more defining relations, namely, that each generator lies in the stable kernel of the underlying peakword. Even though these relations are redundant, they reduce the number of unknowns. Let $\operatorname{mult}_{X}(S)$ denote the multiplicity of a simple $A$-module $S$ in an $A$-module $X$. Then the number of unknowns we have is

$$
\sum_{i=1}^{\operatorname{ncf} M} \operatorname{mult}_{M / J(M)}\left(S_{i}\right) \cdot \operatorname{dim}_{F} \operatorname{StKer}_{N}\left(w_{i}\right),
$$

where $S_{1}, \ldots, S_{\text {ncf } M}$ is a complete set of representatives of the isomorphism classes of the composition factors of $M$.

If moreover the peakwords are chosen with respect to a complete set of representatives of the isomorphism classes of the simple modules appearing as composition factors of $M$ or $N$, then the dimension of $\operatorname{StKer}_{N}\left(w_{i}\right)$ is equal to the multiplicity of $S_{i}$ in $N$, multiplied by the dimension of the splitting field of $S_{i}$ (see Section 3 in [Lux et al. 94]). Hence, we get the following formula for the number of unknowns in this case:

$$
\sum_{i=1}^{\operatorname{ncf}} \operatorname{mult}_{M / J(M)}\left(S_{i}\right) \cdot \operatorname{mult}_{N}\left(S_{i}\right) \cdot \operatorname{dim}_{F} \operatorname{End}_{A}\left(S_{i}\right) .
$$

Note that this case occurs automatically if $M$ contains all simple $A$-modules, or if we are computing endomorphisms, that is, if $M=N$.

\section{THE HOMOMORPHISMS}

We are now able to give an algorithm to compute homomorphism spaces. Its correctness was proved in the previous sections.

\section{Algorithm 5.1. (Homomorphism space.)}

INPUT: $A$-modules $M$ and $N$ given by the action of a generating system $a_{1}, \ldots, a_{\text {ngen } A}$ of $A$; the composition factors $S_{1}, \ldots, S_{\text {ncf } M}$ of $M$; peakwords $w_{1}, \ldots, w_{\text {ncf } M}$ with respect to them; a basis of $J(M)$.

\section{CALCULATION:}

- $i:=1, M_{0}:=\{0\}$, Eqs $:=[], j:=1, \operatorname{SKBas} N:=[]$.

- Do until $M_{i}=M$

- Compute an F-basis SKBasM of $\operatorname{StKer}_{M}\left(w_{j}\right)$.

- For all $x \in S K B a s M$ such that $x \notin M_{i-1}+$ $J(M)$ do

$* v_{i}:=x$.

* Obtain the new part of the spinning basis corresponding to $v_{i}$ and the defining relations corresponding to the extension by calling the $i$-th part of Algorithm 4.1.

* If $S K B a s N=$ [], then

- Let $S K B a s N$ be a basis of $\operatorname{StKer}_{N}\left(w_{j}\right)$.

* Fi.

* Extend Eqs by the linear equations coming from the new defining relations of $M_{i}$, as described in Section 2. 
* Replace Eqs by a maximal subsystem of linearly independent equations of Eqs, using the Gaussian algorithm.

* Increment $i$ by 1 .

- End for.

- Increment $j$ by 1 .

$-\operatorname{SKBasN}:=[]$.

- Od.

- Let $S o l$ be a basis of the solution space of the system of equations Eqs.

- Calculate the matrices for the corresponding basis of $\operatorname{Hom}_{A}(M, N)$ with respect to the spinning basis of $M$ and the original basis of $N$.

OUTPUT: A basis of the homomorphism space $\operatorname{Hom}_{A}(M, N)$.

Remark 5.2. In Algorithm 5.1, we apply a Gaussian algorithm several times. Note that this could be done once at the end, but then we would have an extremely overdetermined system of linear equations, which could lead to memory problems.

Note that the homomorphisms are given with respect to the spinning basis of $M$ and the "original" basis of $N$. If $M=N$, then it is more convenient if the endomorphisms are given with respect to a single basis of $M$ (it is the spinning basis of $M$ ), so a basis transformation has to be performed. Moreover, the homomorphism space is an $F$-algebra in this case, so we might need an algebra generating system of it. This can be achieved by choosing random elements of the algebra and using an "algebra spinning algorithm," which is similar to Algorithm 4.1 for modules. This algorithm is used in the algorithm determining a decomposition of a module into indecomposable direct summands and will be published in a subsequent paper (see [Lux and Szőke 03]).

\section{THE IMPLEMENTATION}

The second author has implemented Algorithm 5.1 in the C-Meat-Axe, now available as a standard program of Version 2.4.0 (see [Ringe 01]). The input of the program mkhom is a list of matrices giving the operation of generators of the algebra $A$ on the modules $M$ and $N$, peakwords for the composition factors of $M$, bases of the stable kernels in $M$ of the peakwords, and a basis of the radical of $M$. The program computes a basis of the homomorphism space $\operatorname{Hom}_{A}(M, N)$ and the base change matrix transforming the original basis of $M$ into the spinning basis of $M$. The homomorphisms are given with respect to the spinning basis of $M$ and the original basis of $N$, or, in case $M=N$, with respect to the spinning basis.

In the following, we give timings for the MAGMA procedure AHom (see Section 3.11 in Chapter "Modules and Lattices" of [Bosma and Cannon 99]) and our program mkhom. The computations were done with MAGMA Version 2.6.

For MAGMA, we used the following script:

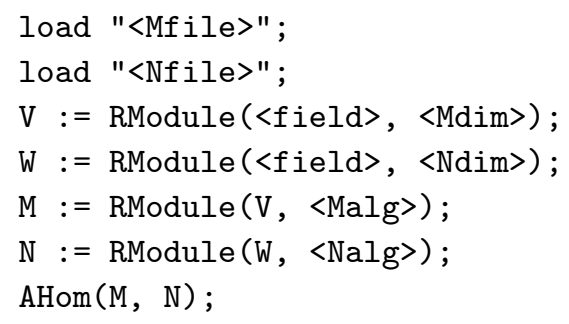

Here, Mfile and Nfile contain the matrix algebras Malg and Nalg over the field field, which are representations of the algebra $A$ describing the source and destination modules $M$ and $N$, respectively.

We used the following C-Meat-Axe procedure:

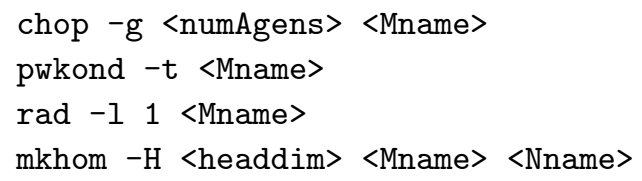

Here, numAgens is the number of generators of the algebra $A$; Mname and Nname are the name of the source and the destination modules $M$ and $N$, respectively; and headdim is the dimension of the head of $M$, given by the C-Meat-Axe program rad.

Since we use the output of the programs chop, pwkond, and rad of the C-Meat-Axe, we give the sum of the running times of all four programs. For the MAGMA procedure, we give the average of three timings. The computations were done on a Pentium II computer with a $400 \mathrm{MHz}$ processor and $1 G B$ main memory under Linux 2.2.10.

In Table 1, we list the following data:

(i) the algebra,

(ii) the number of its generators $(n)$,

(iii) the dimensions of the modules (dim), 


\begin{tabular}{|c|c|c|c|c|c|c|c|c|c|c|}
\hline & \multicolumn{4}{|c|}{$M$} & $N$ & & \multicolumn{2}{c|}{ MAGMA } & \multicolumn{2}{c|}{ C-Meat-Axe } \\
\hline algebra & $n$ & dim & cl & Ll & dim & hom & time & mem & time & mem \\
\hline$c\left(\mathbb{F}_{2} J_{1}\right)$ & 3 & 93 & 44 & 7 & $M$ & 39 & $2.2 s$ & 3.2 & $0.7 s$ & 0.6 \\
\hline$c\left(\mathbb{F}_{4} J_{1}\right)$ & 3 & 93 & 54 & 7 & $M$ & 39 & $4.5 s$ & 3.6 & $1.2 s$ & 0.6 \\
\hline$c\left(\mathbb{F}_{2} J_{2}\right)$ & 5 & 252 & 27 & 13 & $M$ & 8 & $6.5 s$ & 3.9 & $3.9 s$ & 0.7 \\
\hline$c\left(\mathbb{F}_{2} M_{11}\right)$ & 8 & 167 & 167 & 4 & 201 & 2034 & $935 s$ & 30.1 & $222 s$ & 7.2 \\
\hline$c\left(\mathbb{F}_{3} M_{23}\right)$ & 4 & 344 & 169 & 7 & $M$ & 371 & $626 s$ & 35.9 & $73 s$ & 1.5 \\
\hline $\mathbb{F}_{3} G L_{4}(3)$ & 2 & 1596 & 29 & 7 & 361 & 0 & $70 m$ & 23.8 & $74 m$ & 8.3 \\
\hline $\mathbb{F}_{3} G L_{4}(3)$ & 2 & 361 & 10 & 5 & 336 & 0 & $45 s$ & 4.3 & $25 s$ & 1.0 \\
\hline$c\left(\mathbb{F}_{3} H S\right)$ & 6 & 683 & 279 & 5 & $M$ & 1105 & $225 m$ & 380 & $18 m$ & 6.9 \\
\hline$c\left(\mathbb{F}_{3} H S\right)$ & 6 & 683 & 279 & 5 & 775 & 1255 & $244 m$ & 487 & $27 m$ & 9.1 \\
\hline$c\left(\mathbb{F}_{25} H N\right)$ & 2 & 800 & 180 & 19 & $M$ & 146 & $244 m$ & 299 & $48 m$ & 14.5 \\
\hline$c\left(\mathbb{F}_{25} H N\right)$ & 2 & 1564 & 388 & 19 & $M$ & 344 & $?$ & $?$ & $568 m$ & 106 \\
\hline
\end{tabular}

TABLE 1. Timings.

(iv) the composition length (cl) and the Loewy length (Ll) of $M$,

$(v)$ the dimension of the homomorphism space (hom),

(vi) the running times (time) and the memory use (mem) for MAGMA and the C-Meat-Axe (CMA).

For the algebra, a letter $c$ indicates that it is a condensation of the group algebra. In case $M=N$, we write the letter $M$ instead of the dimension of $N$. The memory use is always given in megabytes. All the modules are available on the home page of the first author under http://www.math.arizona.edu/ ${ }^{\sim}$ lux.

In the last example, MAGMA was not able to compute the homomorphism space because it ran out of memory (maximum 1GB) after 1330 minutes.

\section{ACKNOWLEDGMENTS}

This project was partially supported by the Hungarian Scientific Research Grant OTKA number T 034878

\section{REFERENCES}

[Bosma and Cannon 99] W. Bosma and J. Cannon. Handbook of Magma Functions. Sidney: School of Mathematics, University of Sydney, 1999.
[Green et al. 01] E. L. Green, L. S. Heath, and C. A. Struble. "Constructing Homomorphism Spaces and Endomorphism Rings." J. of Symbolic Computation 32:1-2 (2001), 101-117.

[Lux et al. 94] K. Lux, J. Müller, and M. Ringe. "Peakword Condensation and Submodule Lattices: An Application of the Meat-Axe." J. Symbolic Computation 17 (1994), 529-544.

[Lux and Szőke 03] K. M. Lux and M. Szőke. "Decomposing Modules of Finite Dimensional Algebras." In preparation, 2003.

[Lux and Wiegelmann 01] K. Lux and M. Wiegelmann. "Determination of Socle Series using the Condensation Method." J. of Symbolic Computation 31:1-2 (2001), 163-178.

[Parker 84] R. A. Parker. "The Computer Calculation of Modular Characters (the Meat-Axe)." In Computational Group Theory, edited by M. D. Atkinson, pp. 267-274. New York: Academic Press, 1984.

[Ringe 01] M. Ringe. The C-Meat-Axe, Version 2.4.0. RWTH Aachen. Available from World Wide Web (http://www.math.rwth-aachen.de/LDFM/ homes/MTX/download.html), 2001.

[Schneider 90] G. J. A. Schneider. "Computing with Endomorphism Rings of Modular Representations." J. of Symbolic Computation 9 (1990), 607-636.

[Szőke 98] M. Szőke. "Computing Green Correspondents of Weight Modules." PhD diss., Aachener Beiträge zur Mathematik, 1998.

Klaus M. Lux, Department of Mathematics, University of Arizona, 617 North Santa Rita, Post Office Box 210089, Tucson, Arizona 85721 (klux@math.arizona.edu)

Magdolna Szőke, Alfréd Rényi Mathematical Institute, Hungarian Academy of Sciences, P.O.B. 127, 1053 Budapest, Hungary (mszoke@math-inst.hu)

Received March 25, 2003; accepted April 17, 2003. 